\title{
Influence of raceway waviness on the level of vibration in rolling-element bearings
}

\author{
S. ADAMCZAK and P. ZMARZŁY* \\ Faculty of Mechatronics and Mechanical Engineering, Kielce University of Technology, \\ 7 Tysiąclecia Państwa Polskiego Ave., 25-314 Kielce, Poland
}

\begin{abstract}
This paper provides a quantitative analysis of how raceway waviness (RONt) in 6304-type bearings affects their vibration. The waviness of bearing races was measured at the actual points of contact between the balls and the races. The measurements were conducted in the range of 16-50 undulations per revolution (UPR). The bearing vibration was analyzed in three bandwidths of frequency: low (LB) (50 $\div 300 \mathrm{~Hz}$ ), medium MB $(300 \div 1800 \mathrm{~Hz})$ and high HB $(1800 \div 10000 \mathrm{~Hz})$, as well as in the full RMS bandwidth. The paper also presents the procedure used to determine the actual points of contact between the ball and each race to specify the point of waviness measurement. The method of calculation of the contact angle for a ball bearing is also discussed. The Pearson linear correlation coefficients were determined to analyze the relationships between the waviness parameters and the level of vibration. The test results show that an increase in the surface waviness on the inner and outer raceways causes an increase in the vibration level. The influence is most visible for the medium frequency bandwidth.
\end{abstract}

Key words: rolling-element bearings, vibration, waviness, Anderon, technological heredity.

\section{Introduction}

Rolling-element bearings carry loads and minimise the sliding friction by placing rolling elements between two bearing rings (outer and inner raceways). Rolling bearings are the most important mechanical devices, because their quality and reliability often affect the life span and functionality of a whole machine. Defects and faults of bearings can cause unexpected downtime or even irreversible damage to the machine. This is particularly important in cases of large and heavy cylindrical parts, because any interference to such mechanisms is time-consuming and costly $[1,2]$. Therefore, the diagnostics of the technical condition of a rolling-element bearing is very important.

Vibration amplitude is a significant rolling-elements bearings exploitation parameter. One of the factor affecting the vibrations generated by rolling bearings is surface geometry of their races, described by waviness deviation. Analyzing the literature, it can be concluded that a lot of research related to the study of impact of waviness deviation on the vibration amplitude, is based only on simulation [3]. There is a limited number of works focused on experimental research that use rolling elements-bearing commonly available on the market [4]. Furthermore, the waviness measurement method in many research works is not described in detail. They often lack the description of the measuring devices used in research, the method of profile filtration, etc. The authors of [2] have shown that the selection of the appropriate location of waviness profile measurement affects the value of the waviness deviation. Therefore, before measurement of the waviness profile, the contact point of ball and raceways should be deter-

*e-mail: pzmarzly@tu.kielce.pl

Manuscript submitted 2016-07-22, revised 2016-12-23, 2017-01-18 and 2017-01-23, initially accepted for publication 2017-01-27, published in August 2017. mined. Moreover, it should be noted that the bearing industry applied the Anderon unit to analyze the vibrations generated by a rolling-element bearing. The study of the literature revealed that there is a lack of research works describing and applying the Anderon unit. This is very important from the point of view of industrial application. In response to the demands of bearing industry, the authors determined the influence of waviness deviation (measured on the contact point of balls and races) on the vibration expressed by the Anderon unit.

\section{Rolling bearings diagnostic methods}

There are many rolling bearings diagnostic methods [5]. The most commonly used techniques are vibration measurement, acoustic emission (AE) analysis, electrostatic (ES) measurement $[6,7]$, bearing temperature analysis [8], ultrasonic measurement [9], Shock Pulse Method (SPM) [10], wear debris analysis [11], and modal analysis [12]. Despite the fact that there are many techniques to monitor the conditions of rolling-element bearings, the vibration analysis is one of the basic methods to control technical conditions of new bearings. Furthermore, vibration measurement can be applied during the bearing operation. It is worth mentioning that the vibration measurement is a non-destructive testing method, so it is widely used in the machine and bearing industries [13-15]. Vibration analysis allows for real-time monitoring of the condition of rolling-element bearings and taking preventive actions in order to avoid critical machine malfunction. The vibration measuring methods used to monitor the bearing condition were reviewed in $[5,7,16]$. Vibration analysis of rolling-element bearings can be divided into three groups: evaluation based on the general vibration level measurement (e.g. acceleration or velocity of vibra- 
tion), assessment based on the time-frequency vibration signal analysis, and assessment based on specialized signal analysis.

One of the first and basic methods used to analyse the vibration of rolling-element bearings was vibration velocity or acceleration measurement [13]. This kind of measurement can be easily performed using portable measuring devices offered by SKF or FAG companies. Then, the measured values are compared with the reference values presented in the ISO 10816 standard. The main advantage of this method is the possibility of its application to the production cycle for automatic inspection of the rolling-element bearing condition. These procedures allow us to eliminate a defective product directly on the production line.

Another method used to detect defects in ball bearings involves applying a statistical parameter known as the crest factor. The parameter is the ratio of the peak value of the vibration signal to its RMS value in the range of vibration frequencies. The value of the crest factor increases with an increase in the value of the vibration generated by a rolling-element bearing. This method can be easily applied in practice, but it is not suitable for determining the causes of defects occurring in bearings [17]. Furthermore, methods based on the crest factor are very sensitive to an external source of vibration $[17,18]$.

The acoustic emission (AE) method is a non-invasive testing method, because it does not emit signals and therefore does not affect the physical state of the examined bearing [6]. This method is based on the phenomenon of formation of transient elastic waves in the material due to the propagation of, for example, micro scratches, cracks, or corrosion processes in the bearing. Elastic waves generated by a flaw present in the measured rolling-element bearing are emitted in all directions. These waves are usually measured with piezometric transducers. The acoustic emission method is popular tool for monitoring the condition of rolling-element bearing during their operation. Besides, this method is mainly used to analyze the radially-loaded ball bearings operating at low and normal speeds [6]. Despite some drawbacks, the acoustic emission (AE) method is widely used by leading rolling-element bearing manufacturers.

One of the ways of monitoring bearing wear is the application of the electrostatic technique (ES) $[6,19]$. In this method, the electrostatic charge sensor can be located directly on the bearing housing. When a charged particle passes the electrostatic sensor face, an opposing charge will be included on the sensor surface. Consequently, the electrons in the sensor will redistribute, in order to balance the additional charge in the vicinity of the sensor face. As a result of this, current flow will be generated and measured [6]. This type of measurement allows us to detect defects at an early stage. This is the main advantage of this method. In measurement based on the electrostatic technique, the factors indicating upcoming bearing defects are directly monitored, rather than just their critical effects, such as acceleration of vibration or rapid rise in the bearing temperature. Consequently, this method is usually used in active control systems to monitor the condition of an operating rolling-element bearing, therefore being very popular in the bearing or machine industries.

A large group of predictive rolling-element bearings health monitoring tools are the methods based on the vibration signal analysis. The main advantage of these methods is the possibility to detect and separate external vibration signals or background noise. One of the first and most popular tools used to assess the rolling-element bearings vibration signal was the spectral analysis based on the fast Fourier transform (FFT) [5, 16]. This signal processing technique has been used on a large scale for converting the time domain contents into the frequency spectrum. The most important asset of this method is the simplicity of application and popularity of Fourier transformation. But the main drawback of this technique is the difficulty of detecting some bearing failures, because external vibrations affect the vibration signal. What is more, harmonic analysis of a vibration signal using FFT can cause false assessment of the technical condition of rolling-elements bearings. Consequently, nowadays, beside the FFT signal analysis, other transformations have been applied, for example the wavelet transform [16, 20, 21], the short-time Fourier transform (STFT), and the Haar transform.

Another interesting technique used to analyse a vibration signal is the envelope method. The envelope technique utilizes the idea of detecting the fault impulses that are amplified by structural resonance. Based on the information related to the bearing geometry, the number of rolling elements and the rotational speed of the inner race relative to the outer race, it is possible to calculate the characteristic frequency harmonic of the signal obtained from the individual parts of the rolling-element bearing. The envelope method allows us to determine which element of the bearing generates a higher vibration amplitude and thus, it allows us to conclude that it is damaged [22]. A disadvantage of this method is that it is difficult to detect faults based on the poor quality of a vibration signal.

The vibration amplitudes generated by a rolling-element bearing, and hence its quality, depend on many factors, including the internal clearance [23], the grease type [4, 11], the load type [24], as well as the surface topography of the raceways and balls. However, a waviness deviation of cylindrical surfaces plays a significant role [4, 25-27]. The waviness deviations of the active surfaces of bearing can be caused by, among others, an improper manufacturing technology, or abrasive and fatigue wear. In the case of excessive values of waviness, the contact force between the rolling elements and races increases, resulting in an increased amplitude of the vibration, and thus a rapid wear of the bearing. Furthermore, the authors of the paper [4] indicate that the variation of the oil film in thrust ball bearings is related to the raceway waviness.

The measurement of the waviness profile of bearing raceways is a complicated and invasive procedure. Before the measurement, it is necessary to remove the cage in order to open the bearing and get access to surfaces of raceways and rolling elements. In many cases, after the bearing disassembly, its reuse is difficult or even impossible. Accordingly, the analysis of raceways or balls waviness deviations and associating it with the vibrations generated by rolling-element bearings is a crucial aspect. The application of this analysis will allow us to predict the approximate values of waviness deviation of rolling-element bearing parts based on the vibration level, measured using non-invasive measuring methods.

There are many papers analyzing the effect of rolling-element bearing waviness on the vibration level, but in most cases, 
it is not specified what range of UPR (undulation per revolution) is considered. Moreover, the authors of these papers usually do not define what frequency of vibration is examined, or the vibration analysis is carried out in full-range RMS. It should be noted that in most research works it is not specified how waviness deviations of bearing raceways or balls were measured. Some simplification is assumed, namely that waviness deviation is the same throughout the raceway of the bearing. The experience shows that form errors of the rolling-element bearing surfaces may have different values that are dependent on the machining process. Therefore, the waviness profile should be measured at the actual point of contact between the balls and the raceways. In this study, the contact points for each examined rolling-element bearing were determined. Only then was the waviness measured. It is important to note that most papers describe simulation studies $[25,28]$. However, the research on ball bearings available on the market is limited.

This paper discusses a study carried out to determine the influence of waviness of rolling-element bearing raceways on the generated vibration amplitudes. Waviness deviations were examined in the range of 16-50 UPR. The vibration analysis was carried out in three frequency bandwidths: low bandwidth LB, medium bandwidth MB, and high bandwidth HB. Moreover, the vibration results were presented in the full RMS range and the harmonic analysis of vibration signal was carried out. Additionally, the paper shows a procedure for determining the actual points of contact between the balls and the inner and outer raceways.

\section{Experiment}

The experiments were carried out at the Laboratory of Rolling-Element Bearings at the Kielce University of Technology. Fifty open single-row deep groove ball bearings (type 6304) were used in the tests. Fig. 1 shows the examination procedure.
3.1. Vibration measurement. The vibrations generated by rolling-element bearings were measured using an Anderometer measuring device developed at the Kielce University of Technology. A majority of vibration measurement tools are equipped with a probe used to measure the vibration acceleration. An important characteristic of the Anderometer measuring system is the application of a vibration velocity probe. Deployment of this kind of sensor requires significantly less pre-load of velocity probe. Furthermore, a probe operating in a function of velocity can work at low signal magnification and can measure a low frequency $(30 \mathrm{~Hz})$. Detailed description of the structure and the principle of operation of the Anderometer are presented in [29]. The rolling-element bearings were examined at a rotational speed of $1800 \mathrm{~min}^{-1}$ in accordance with the recommendations presented in the ISO 15242-1:2004 standard. In order to reset the internal clearance during vibration measurement, the bearings were examined under axial load. The vibration analysis was carried out in three frequency bandwidths: the low bandwidth (LB) 50 to $300 \mathrm{~Hz}$, the medium bandwidth (MB) 300 to $1800 \mathrm{~Hz}$, and the high bandwidth (HB) from 1800 to $10000 \mathrm{~Hz}$. In addition, the results of the vibration measurements were presented in the full frequency range (RMS).

The vibrations generated by the examined rolling-element bearings are expressed with the Anderon unit, which is commonly used in the bearing industry. This unit was established in the United States in the 1940s. One Anderon equals approximately $7.698 \mu \mathrm{m} / \mathrm{s}$. The Anderon unit can be defined by (1):

$$
A=\frac{V}{n \sqrt{N}} \text { (Anderon) }
$$

where:

$$
\begin{aligned}
& V-\text { vibration velocity }(\mu \mathrm{inch} / \mathrm{rad}), \\
& n-\operatorname{radian}(\mathrm{rad} / \mathrm{s}), \\
& N-\text { number of octave. }
\end{aligned}
$$

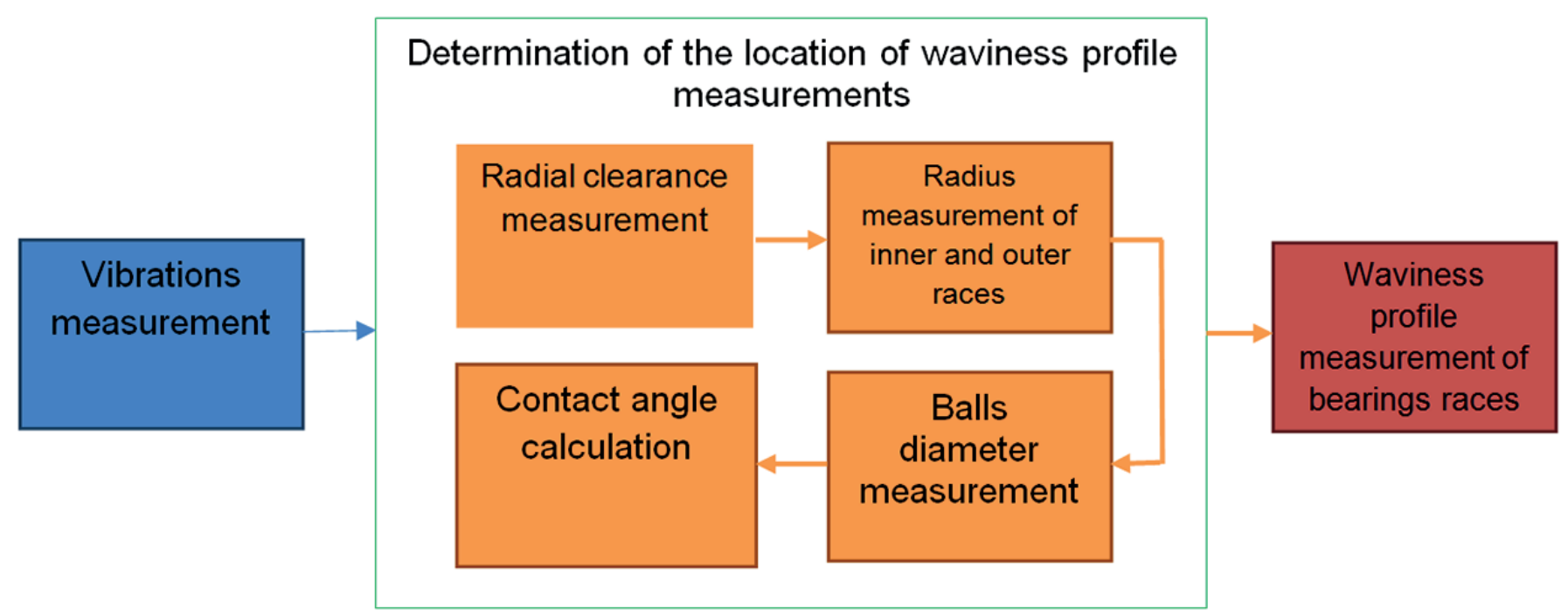

Fig. 1. Diagram of the examination procedure 


\subsection{Determining the contact point of ball and raceways.}

During the operation of the rolling bearing, the ball is in contact with the surfaces of the inner and outer raceways at points A and B (Fig. 2). Analyzing the machining process of the bearing surfaces, it can be noted that the bearing raceways may have different values of waviness deviations in different locations. Therefore, it is desirable to analyze the waviness profile in the real contact point of the bearing ball and raceways surfaces (points A and B).

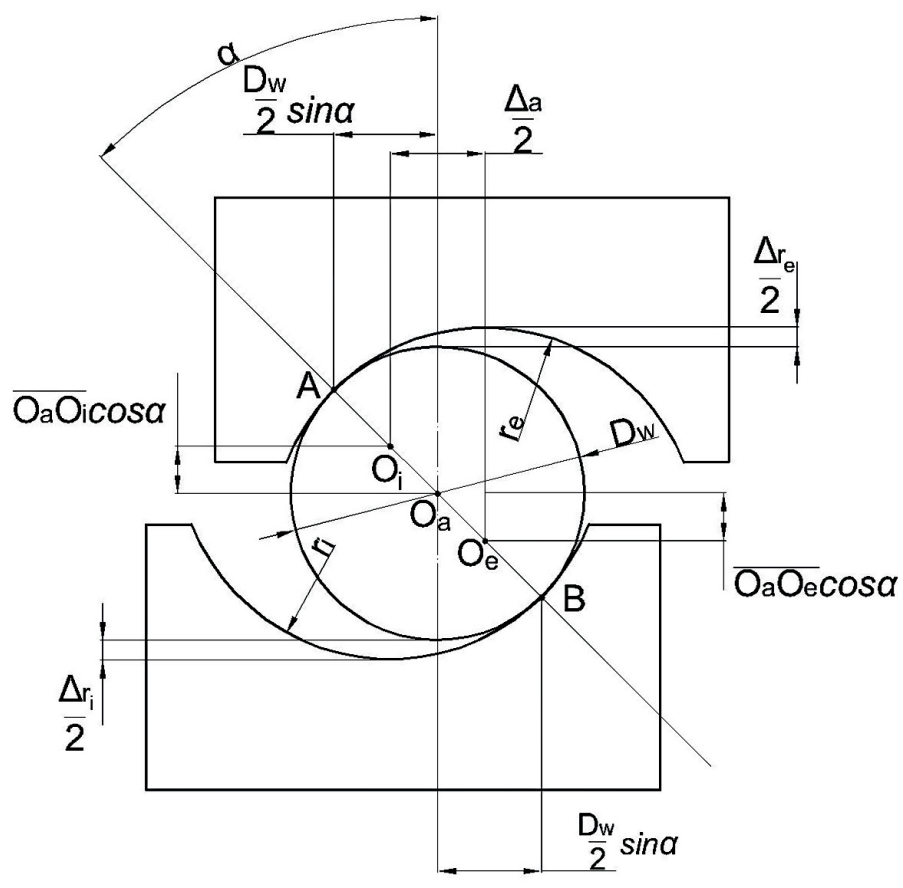

Fig. 2. Geometrical relationship between the ball and the raceways for deep groove ball bearings, where: $\mathrm{A}$ - contact point of ball and outer race, $\mathrm{B}$ - point of ball and inner race, $\alpha$ - contact angle $\left(^{\circ}\right), O_{a}$ - ball centre, $O_{e}$ - center of the groove curvature, outer ring, $O_{i}$ - center of the groove curvature, inner ring, $D_{w}$ - ball diameter $(\mathrm{mm}), r_{e}$ - radius of the outer ring groove $(\mathrm{mm}), r_{i}$ - radius of the inner ring groove $(\mathrm{mm})$,

$\Delta_{r}$ - radial clearance $(\mathrm{mm})$, and $\Delta_{a}$ - axial clearance $(\mathrm{mm})$

Analyzing Fig. 2 it can be noted that to determine the contact point of the ball and races, relations based on values of contact angle $\alpha$ and ball diameter $\mathrm{D}_{\mathrm{w}}$ can be used. The contact angle $\alpha$ is define as the angle between the straight line connecting the contact points of the ball and the races in the radial plane, along which the load is transmitted from one race to another (Fig. 2). The rolling bearing contact angle can either be determined experimentally or calculated based on geometrical relationships. One of the methods used to determine the contact angle has been proposed by the NSK company in [30]. In order to calculate the contact angle, the detailed geometrical relations of ball and raceways have been analyzed (Fig. 2). by (2):

Analyzing Fig. 2, the radial clearance $\Delta_{r}$ can be described

$$
\Delta_{r}=\Delta r_{e}+\Delta r_{i}
$$

Next, the geometric relationships were used to determine radial clearance $\Delta_{r}(3)$ and axial clearance $\Delta_{a}(4)$ :

$$
\begin{gathered}
\Delta_{r}=2(1-\cos \alpha)\left(r_{e}+r_{i}-D_{w}\right), \\
\Delta_{a}=2 \sin \alpha\left(r_{e}+r_{i}-D_{w}\right),
\end{gathered}
$$

where:

$$
\cot \frac{\alpha}{2}=\frac{\Delta_{a}}{\Delta_{r}}
$$

then:

$$
\Delta_{a}=2\left(r_{e}+r_{i}-D_{w}\right)^{\frac{1}{2}} \Delta_{r}^{\frac{1}{2}} .
$$

Basing on (2-6), the contact angle $\alpha$ of deep-groove ball bearings can be defined by (7):

$$
\alpha=\cos ^{-1}\left(\frac{r_{e}+r_{i}-D_{w}-\Delta_{r} / 2}{r_{e}+r_{i}-D_{w}}\right)=\sin ^{-1}\left(\frac{\Delta_{a} / 2}{r_{e}+r_{i}-D_{w}}\right) .
$$

Considering (7), we can notice that the value of the contact angle depends on the radial $\Delta_{r}$ or axial $\Delta_{a}$ clearance, the radius of the inner $r_{i}$ and outer $r_{e}$ races, and ball diameters $D_{w}$. Therefore, by measuring the rolling-element bearing internal clearance and analyzing the surface geometry of the raceways and the balls, the contact angle $\alpha$ can be easily calculated.

The first step of the contact angle determination procedure was radial clearance measurement of each rolling-element bearing using the measuring device shown in Fig. 3a. The internal clearance was measured under a load of $50 \mathrm{~N}$. After the clearance measurement, the next step was to disassemble the bearings in order to get access to the surfaces of the raceways and the balls. For this purpose, a hydraulic press was used to remove the rivets from the cage. After that, the balls and the outer and inner rings were separated.

Next, the radii of the raceways were measured using a measuring device presented in Fig. 3b. This measuring system is equipped with two independent test heads. One is applied to measure roundness and waviness profiles along the perimeter of the bearing raceways, and the other measuring head is used to measure surface roughness, profile deviation, and the raceway radii. Analyzing (7), we can see that it is necessary to obtain the radius of the inner and outer raceways to determine the contact angle. Therefore, the second measuring head was used in the tests.

During the measurement process, which is performed in the polar coordinates, the measuring head moves along the arc of a specified radius. This solution allows us to facilitate the calculation procedures, because each change in the head position is defined as a change in the radius in relation to the standard radius in the selected rotation axis. Furthermore, the measuring head is always located in the direction perpendicular to the measurement plane, thereby it is always at the same contact point. The analysis of the measurement data and calculation of the radii of the bearing raceways was performed using BEFON software developed at the Kielce University of Technology. 


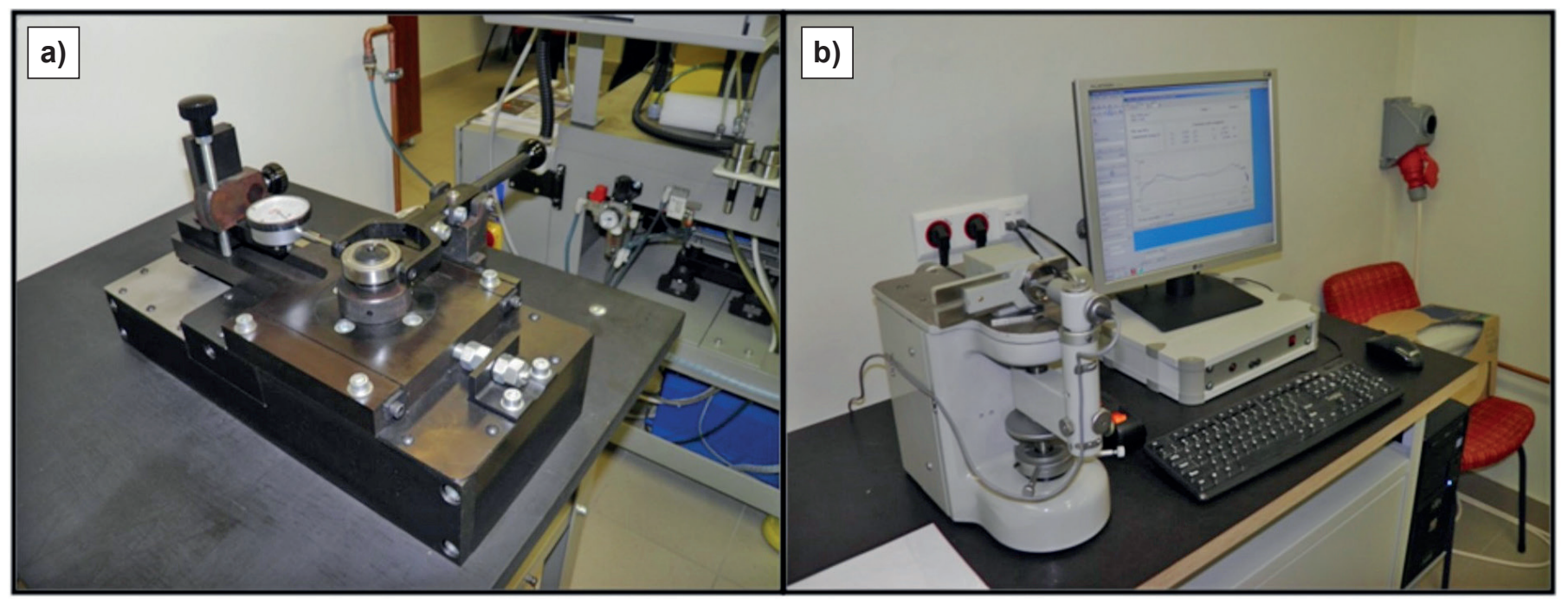

Fig. 3. Measuring systems used in the tests: a) device used to measure radial clearance, b) device used to measure surface roughness, profile deviation, and raceway radius

The last step of the procedure used to calculate the contact angle was to measure the ball diameters $D_{w}$ using a KLM 60 metroscope produced by Steinmeyer Company. Based on the obtained results, the contact angle $\alpha$ was calculated using (7). Then, using the calculated values of the contact angle, the points of contact between the ball and bearing raceways were defined (points A and B in Fig. 4). These contact point define the location of waviness profile measurement.
3.3. Waviness profile measurement. The waviness of the inner and outer bearing raceways was measured using a Talyrond 365 measuring device, located in the Laboratory of Computer Measurements of Geometric Quantities at the Kielce University of Technology. The Talyrond 365 is a high-precision instrument based on the radial change method known as the non-reference method. This measuring system is equipped with a gauge head, which allows a maximum resolution of $1.3 \mathrm{~nm}$ (for the range
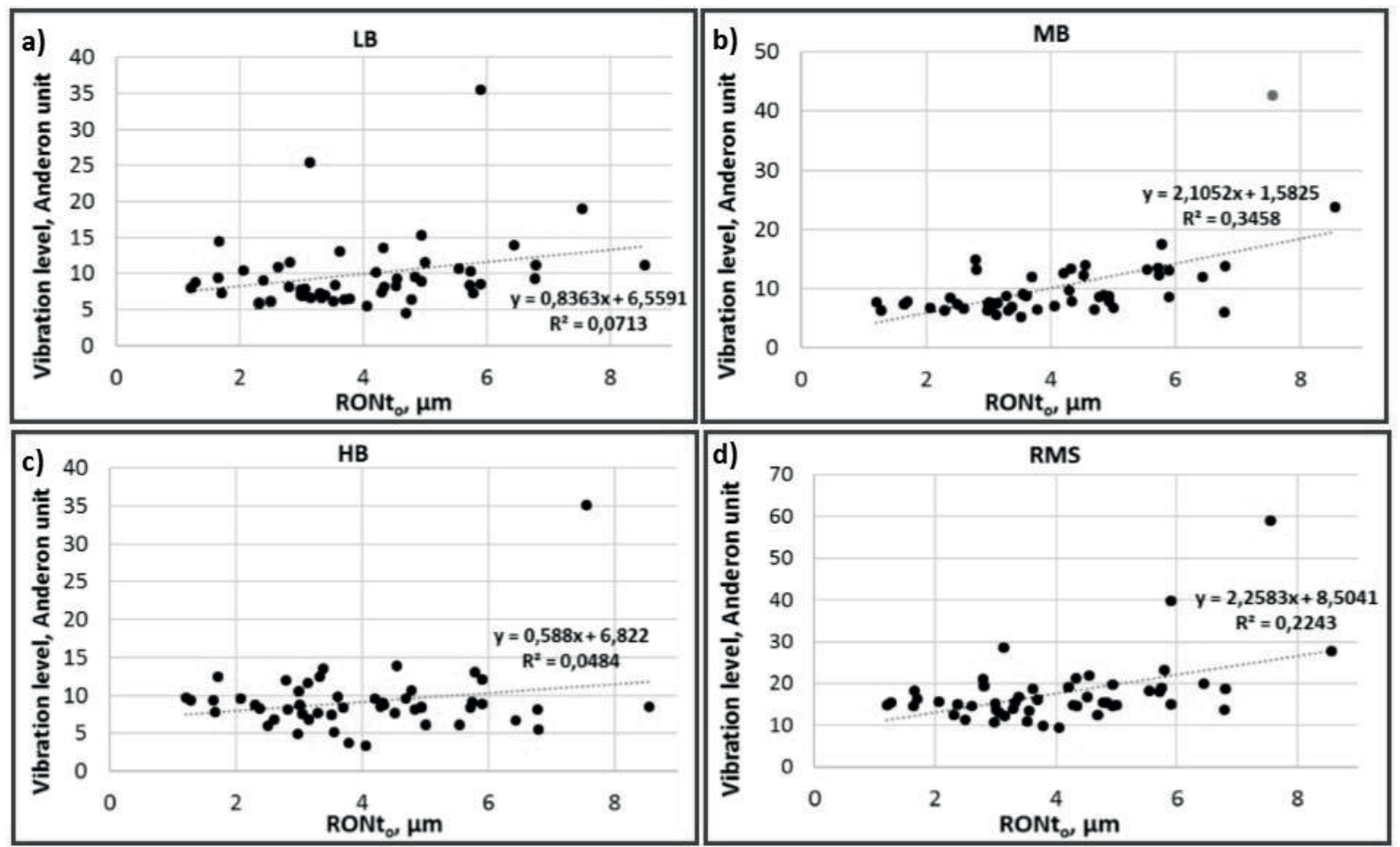

Fig. 4. Relationships between the vibration generated by bearings and waviness deviation $\mathrm{RONt}_{\mathrm{o}}$ of the outer raceways: a) LB - low frequency bandwidth, b) MB - medium frequency bandwidth, c) HB - high frequency bandwidth, d) RMS - full frequency bandwidth 
of $0.08 \mathrm{~mm}$ ). Radial and axial limit of error for Talyrond 365 is $\pm(0.02 \mu \mathrm{m}+0.0003 \mu \mathrm{m} / \mathrm{mm})$. The position of the gauge head relative to the measured object is controlled by an incremental encoder with an accuracy of $\pm 0.5 \mu \mathrm{m}$. This allows to measure waviness at the calculated point of contact between the rolling-element bearing ball and the raceways. The Talyrond 365 is equipped with Ultra Roundness 5.17 software, which can be used to control the measuring procedure and to analyze the geometric structure of the cylindrical surfaces.

In order to analyze the reproducibility of the measuring system, the waviness deviations of one race were measured thirty times. Then, the mean deviation $s$ was calculated to equal $\mathrm{s}=0.008 \mu \mathrm{m}$.

The parameter used in the study was the waviness deviation RONt, which was calculated based on the last square circle (LSC) in the range 16-50 UPR (undulations per revolution). The measured profiles were filtered using a Gaussian filter. At the beginning, the waviness deviations of inner raceways were measured at the calculated contact points. The same measuring procedure was used for the outer raceways.

\section{Results and discussion}

The results of the examined rolling-element bearings were grouped according to the values of the waviness deviation RONt (from lowest to highest). A preliminary analysis of the measurement results indicates that waviness deviations for the outer raceways are in the range of $\mathrm{RONt}_{0} \epsilon<1.21 \mu \mathrm{m}, 8.56 \mu \mathrm{m}>$; however, for the inner races in the range of $\mathrm{RONt}_{\mathrm{i}} \epsilon<1.30 \mu \mathrm{m}$, $6.93 \mu \mathrm{m}>$. In addition, in order to determine the relationship between the measured waviness deviations of bearing raceways and the vibration level generated by rolling-element bearings, the Pearson linear correlation coefficients were calculated [31]. Subsequently, the correlation between the analyzed parameters was determined based on the rules provided by I.P. Guilford [31], which are presented in Table 1 . The vibration level generated by the examined rolling bearings was described in the Anderon unit that equals approximately $7.698 \mu \mathrm{m} / \mathrm{s}$.

Table 1

Correlation and relationships between parameters based on the classification by I.P. Guilford [28]

\begin{tabular}{|c|c|c|}
\hline Correlation coefficient $\boldsymbol{r}$ & Correlation & Correlation relationship \\
\hline below 0.20 & weak & almost meaningless \\
\hline $0.20-0.40$ & low & pronounced, but low \\
\hline $0.40-0.70$ & moderate & significant \\
\hline $0.70-0.90$ & high & strong \\
\hline $0.90-1.00$ & very high & certain \\
\hline
\end{tabular}

4.1. Results for the outer raceways. The results obtained for the outer raceways of 6304-type bearings are shown in Fig. 4. The diagrams present the relationships between the vibration generated by the bearings and the waviness deviation of the outer raceways (Fig. 4). The vibration analysis was performed for three vibration frequency ranges (low LB, medium $\mathrm{MB}$, and high $\mathrm{HB}$ ) and full range RMS. In order to facilitate the interpretation of the measuring results presented in Fig. 4, the trend lines on the charts were plotted with a regression equation and the coefficient of determination $\mathrm{R}^{2}$. Additionally, Table 2 shows the correlation of analyzed parameters based on the Pearson linear correlation coefficients.

\section{Table 2}

Correlation between the vibration generated by 6304-type bearings and the waviness deviations $\mathrm{RONt}_{\mathrm{o}}$ of the outer raceways

\begin{tabular}{|c|c|c|c|c|}
\hline $\begin{array}{c}\text { Vibration } \\
\text { frequency } \\
\text { bandwidth }\end{array}$ & RMS & LB & MB & HB \\
\hline $\begin{array}{c}\text { Pearson } \\
\text { linear } \\
\text { correlation } \\
\text { coefficient }\end{array}$ & $\mathrm{r}=0.47$ & $\mathrm{r}=0.27$ & $\mathrm{r}=0.59$ & $\mathrm{r}=0.22$ \\
\hline $\begin{array}{c}\text { Correlation } \\
\text { relationship }\end{array}$ & significant & $\begin{array}{c}\text { pronounce, } \\
\text { but low }\end{array}$ & significant & $\begin{array}{c}\text { pronounce, } \\
\text { but low }\end{array}$ \\
\hline
\end{tabular}

Analyzing the research results obtained for the outer raceways, it can be concluded that an increase in the value of waviness deviation $\mathrm{RONt}_{\mathrm{o}}$ measured in the range 16-50 UPR causes an increase in the vibration amplitudes for all frequency bandwidths (Fig. 4). Considering the trend lines shown in the diagrams in Fig. 4, it can be seen that the largest increase in vibration is for the medium frequency bandwidth MB (Fig. 4b), but the lowest for the high vibration frequency bandwidth HB (Fig. 4c). It is confirmed by the calculated Pearson linear correlation coefficients presented in Table 2. As we can see in this table, the highest value of the Pearson coefficient was calculated for the medium frequency bandwidth $\mathrm{MB}(\mathrm{r}=0.59)$. The classification presented in the I.P. Guilford Table (Table 1) indicates a significant correlation relationship between waviness deviation measured on the outer raceways and the vibration level analyzed in the medium frequency bandwidth, i.e. from $300 \mathrm{~Hz}$ to $1800 \mathrm{~Hz}$. While the lowest value of the Pearson linear correlation coefficient was calculated for the high frequency bandwidth HB $(r=0.22)$, which shows a pronounced correlation, but a low one.

4.2. Results for the inner raceways. Like in the previous subsection, Fig. 5 shows the relationship between the vibration and waviness $\left(\mathrm{RONt}_{\mathrm{i}}\right)$ of the inner raceways. Table 3 presents the correlation between the analyzed parameter.

The test results obtained for the inner raceways are similar to the measurement results achieved for the outer raceways. In this case, an increase in the waviness of the inner raceways causes a rise in the vibration level, which was analyzed in all frequency bandwidths. The largest growth was observed for the medium frequency bandwidth $\mathrm{MB}$, and the lowest for the low frequency bandwidth LB (Fig. 5). The Pearson linear correlation coefficients calculated for the inner races (Table 3 ) indicate 

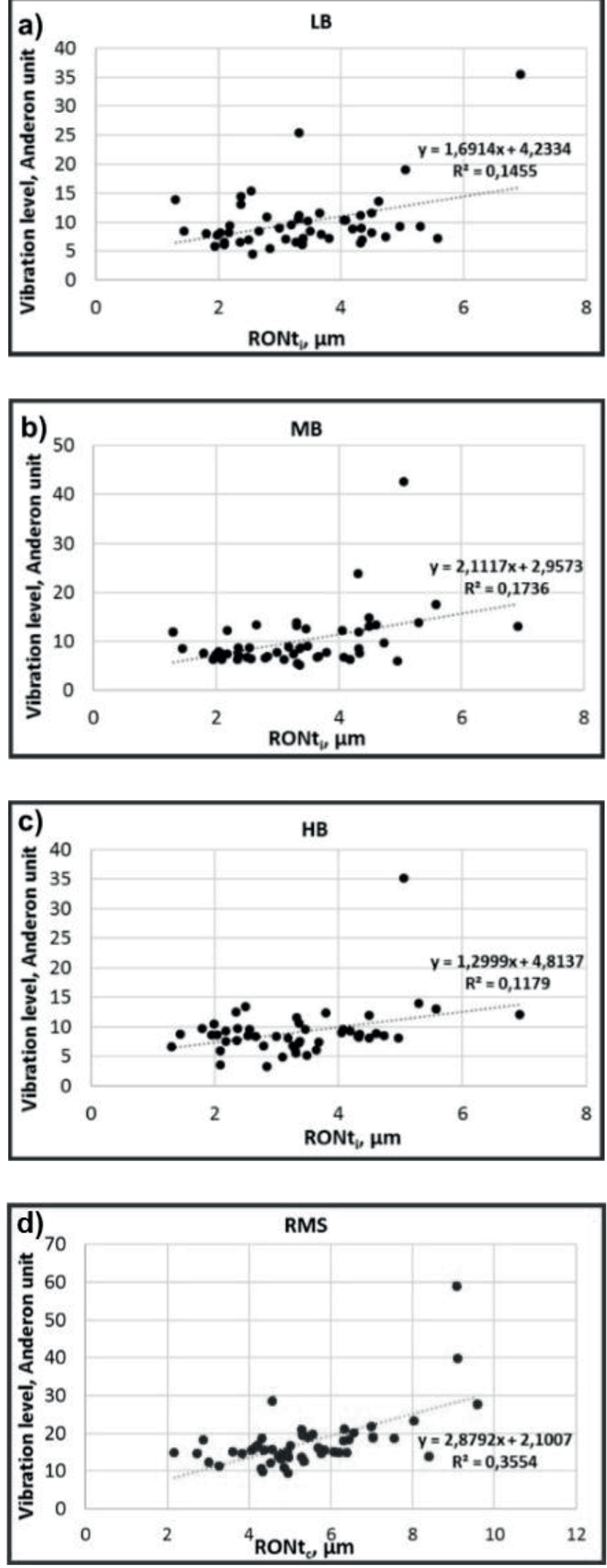

Fig. 5. Relationships between the vibration generated by bearings and waviness deviation RONt of the inner raceways: a) LB - low frequency bandwidth, b) MB - medium frequency bandwidth, c) HB - high frequency bandwidth, d) RMS - full frequency bandwidth a significant correlation for the medium frequency bandwidth MB $(r=0.42)$ and a pronounced, but low correlation for the high and low vibration frequency bandwidths.

Table 3

Correlation between the vibration generated by 6304-type bearings and the waviness deviations $\mathrm{RONt}_{\mathrm{i}}$ of the inner raceways

\begin{tabular}{|c|c|c|c|c|}
\hline $\begin{array}{c}\text { Vibration } \\
\text { frequency } \\
\text { bandwidth }\end{array}$ & RMS & LB & MB & HB \\
\hline $\begin{array}{c}\text { Pearson } \\
\text { linear } \\
\text { correlation } \\
\text { coefficient }\end{array}$ & $\mathrm{r}=0.49$ & $\mathrm{r}=0.38$ & $\mathrm{r}=0.42$ & $\mathrm{r}=0.34$ \\
\hline $\begin{array}{c}\text { Correlation } \\
\text { relationship }\end{array}$ & significant & $\begin{array}{c}\text { pronounce, } \\
\text { but low }\end{array}$ & significant & $\begin{array}{c}\text { pronounce, } \\
\text { but low }\end{array}$ \\
\hline
\end{tabular}

4.3. Comprehensive results. Vibration generated by rolling bearings result from waviness deviation of both inner and outer raceways. Therefore, in order to carry out a detailed analysis, the waviness deviations for inner race $\left(\mathrm{RONt}_{\mathrm{i}}\right)$ and outer race $\left(\mathrm{RONt}_{\mathrm{o}}\right.$ ) were summed for each rolling-elements bearing using (8):

$$
R O N t_{c}=\sqrt{R O N t_{i}^{2}+R O N t_{o}^{2}} .
$$

Similarly as for the results presented in subsections 4.2 and 4.3, the calculation results were presented in diagrams in Fig. 6 and Table 4.

Table 4

Correlation between the vibration generated by 6304-type bearings and the sum of waviness deviations $\mathrm{RONt}_{\mathrm{c}}$ of the inner and outer raceways

\begin{tabular}{|c|c|c|c|c|}
\hline $\begin{array}{c}\text { Vibration } \\
\text { frequency } \\
\text { bandwidth }\end{array}$ & RMS & LB & MB & HB \\
\hline $\begin{array}{c}\text { Pearson } \\
\text { linear } \\
\text { correlation } \\
\text { coefficient }\end{array}$ & $\mathrm{r}=0.60$ & $\mathrm{r}=0.39$ & $\mathrm{r}=0.65$ & $\mathrm{r}=0.32$ \\
\hline $\begin{array}{c}\text { Correlation } \\
\text { relationship }\end{array}$ & significant & $\begin{array}{c}\text { pronounce, } \\
\text { but low }\end{array}$ & significant & $\begin{array}{c}\text { pronounce, } \\
\text { but low }\end{array}$ \\
\hline
\end{tabular}

Analyzing the sum of waviness deviation calculated for inner and outer raceways, it can be concluded that the most visible impact of total waviness deviation $\mathrm{RONt}_{\mathrm{c}}$ on amplitude of vibration is noticed for medium frequency bandwidth MB (Fig. 6c). The Pearson linear correlation obtained for MB reached the highest values out of all calculated $(\mathrm{r}=0.65)$. The lowest correlation between deviation $\mathrm{RONt}_{\mathrm{c}}$ and vibration frequency band has been received for low frequency bandwidth LB. The correlation obtained for the sum of waviness deviation $\mathrm{RONt}_{\mathrm{c}}$ and vibration amplitudes is largely consistent with the correlation calculated separately for inner and outer raceways. 

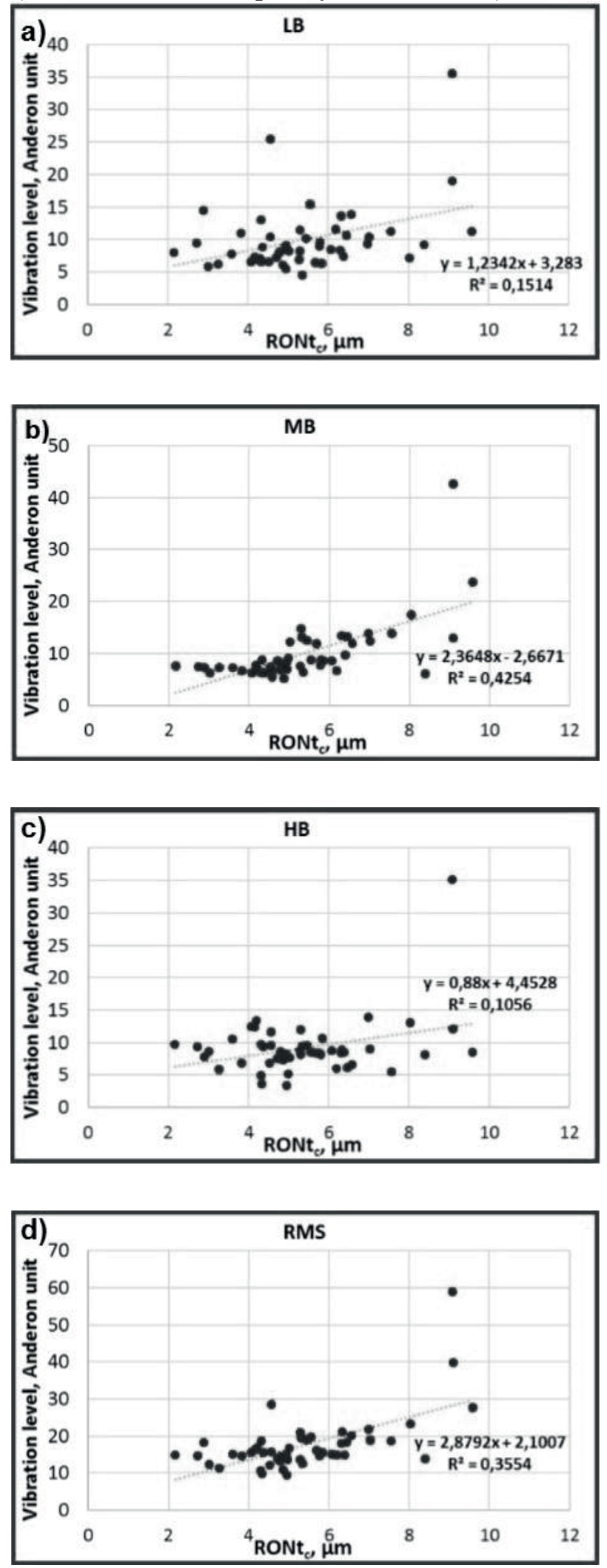

Fig. 6. Relationships between the vibration generated by bearings and sum of waviness deviation $\mathrm{RONt}_{\mathrm{c}}$ of the inner and outer raceways: a) LB - low frequency bandwidth, b) $\mathrm{MB}$ - medium frequency bandwidth, c) HB - high frequency bandwidth, d) RMS - full frequency bandwidth
Additionally, in the paper, the research results for the exemplary 6304-type rolling-element bearing have been presented. Fig. 7 shows the waviness profile of inner and outer races mea-
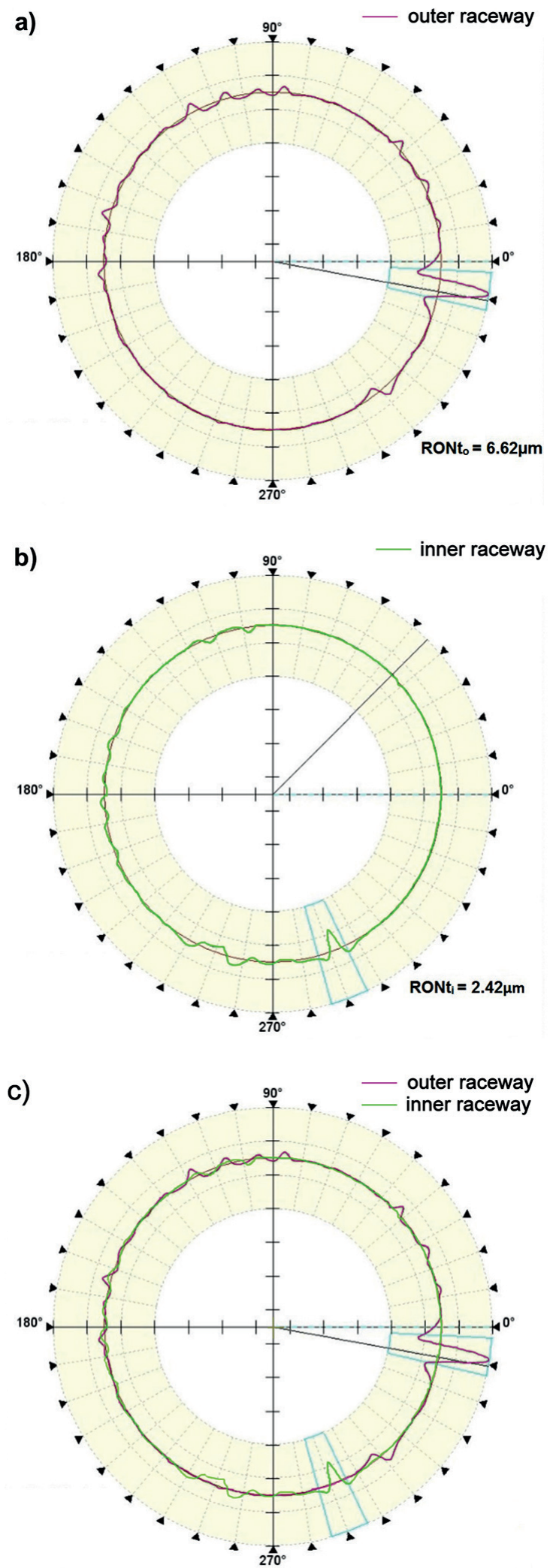

Fig. 7. Waviness profiles of: a) outer raceway, b) inner raceway, c) superimposed profiles 


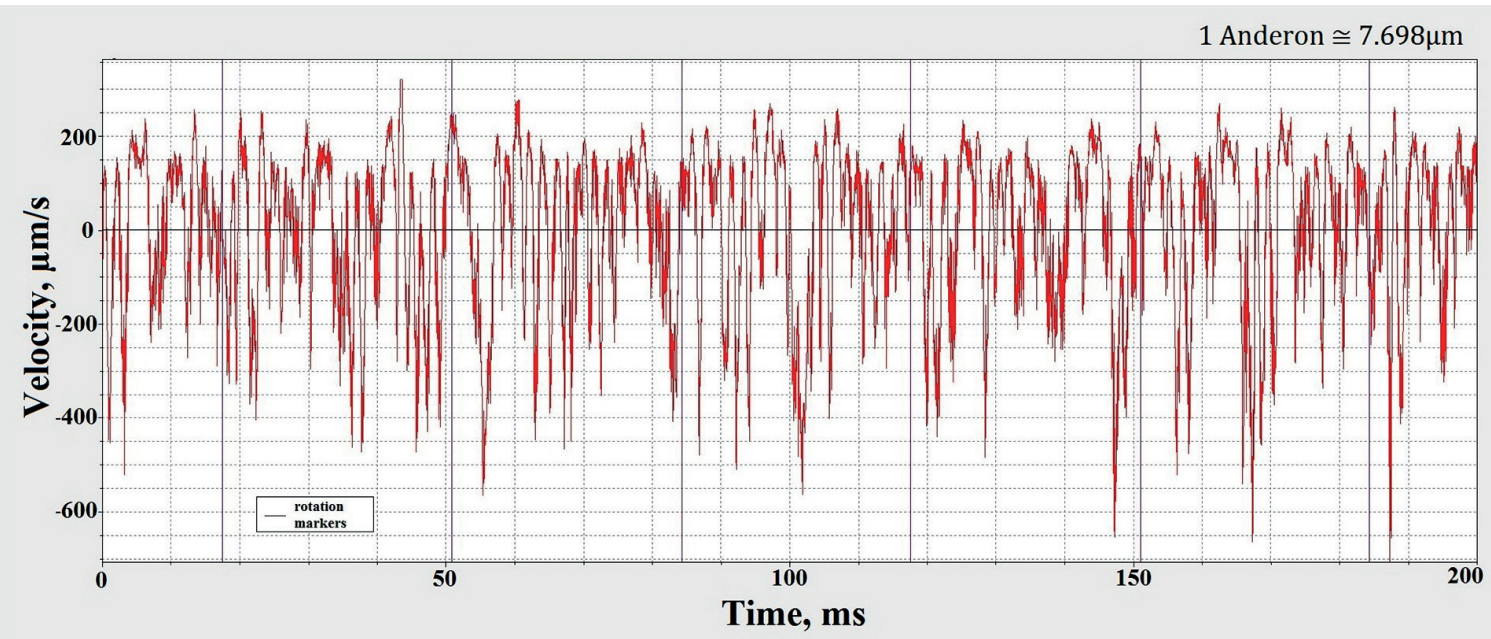

Fig. 8. Time history of vibration velocity for examined rolling-element bearings

sured using a Talyrond 365 device. Analyzing Fig. 7, we can see that the waviness deviations calculated for outer raceway equals $\mathrm{RONt}_{\mathrm{o}}=6.62 \mu \mathrm{m}$, and for the inner raceway it equals $\mathrm{RONt}_{\mathrm{i}}=2.42 \mu \mathrm{m}$. These results show that the surface quality of the bearing's outer race in comparison to the inner race is worse. It may be the result incorrect manufacturing process, for example from a periodic vibration of the grinding wheel spindle.

In order to perform a detailed analysis of research results, in Fig. 8 there was presented a graph of time history of vibration velocity of the examined deep groove ball bearing. The vibration velocity was expressed in $\mu \mathrm{m} / \mathrm{s}$. It should be noted that one Anderon equals approximately $7.698 \mu \mathrm{m} / \mathrm{s}$. In addition, a harmonic analysis of the vibration signal was carried out. Each bearing's element has a characteristic rotational frequency. When fundamental fault frequencies of the bearing's components are known, it is possible to determine which element of the bearing generates high vibration frequency value. In consequence, this may indicate that it is damaged [32]. Fig. 9 shows the magnitude spectrum of the vibration signal with markers that present characteristic frequencies of the bearing's components. Based on research results, we indicate that above $1000 \mathrm{~Hz}$ the frequency values are very low, and therefore the graph shows the spectrum in frequency range $0 \div 1000 \mathrm{~Hz}$.

Analyzing the amplitude spectrum of vibration velocity presented in Fig. 9, the characteristic peaks can be observed. A few times, the outstanding amplitudes can be caused by ball defects, b-stability or multi-stability, or contact resonance. The green markers on the graph present the outer race defect frequency, red markers show the inner race defect frequency, and light blue markers represent the rolling element defect frequency. Furthermore, the brown markers represent the bearing's natural frequencies. As can be seen in Fig. 9, the high peaks of magnitude spectrum overlap with the green marks, which indicate defect of outer bearing's raceway. Furthermore, the inner race defect frequency overlaps with other amplitudes, but their values are low. The results obtained for amplitude spectrum

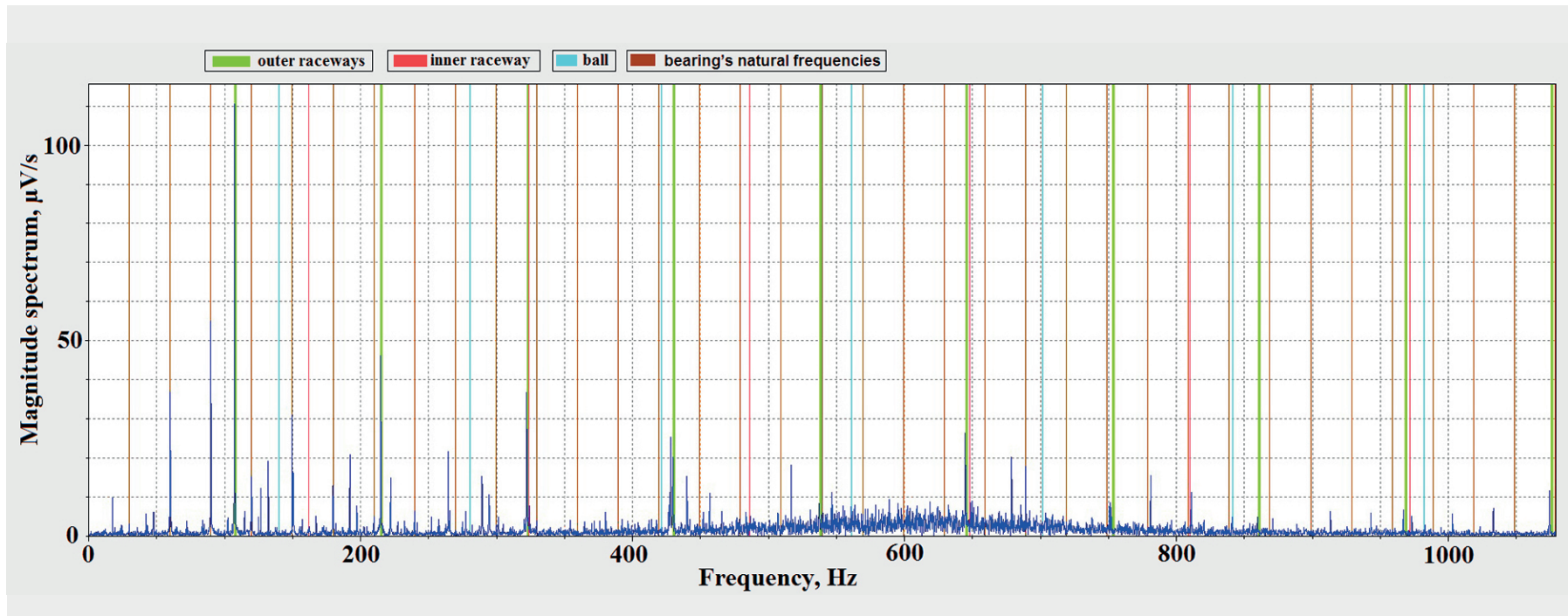

Fig. 9. Amplitude spectrum of vibration velocity for the examined rolling-element bearing 
analysis of the bearing's vibration velocity correspond to the waviness measuring results presented in Fig. 7. The value of waviness deviation $\mathrm{RONt}_{\mathrm{o}}$ measured on the outer raceway is twice as high as waviness deviation $\mathrm{RONt}_{\mathrm{i}}$ obtained for the inner raceway. Based on harmonic analysis of vibration signal, it can be concluded that balls do not affect vibration amplitudes. To sum up, a detailed analysis of vibration amplitudes and geometry of exemplary rolling-element bearing confirms that the waviness deviation of bearing races affects the vibration generated by rolling bearings.

\section{Summary and conclusion}

The intensive development of modern technology requires the bearing manufacturers to increase the quality of their products. It would not be possible without the application of the modern research base. In response to a local market demand, a modern laboratory for testing rolling-element bearings was opened at the Kielce University of Technology.

One of the basic predictive health-monitoring methods of rolling-element bearings is vibration measurement. Based on vibration measurement results, it is possible to evaluate the condition of rolling-element bearings during their operation. The amplitude of vibration generated by bearings is affected by many factors. One of them is the waviness of the inner and outer raceways.

The main aim of the study presented in this paper was to quantitatively analyze the influence of the waviness (RONt) of the inner and outer raceways on the vibration amplitudes generated in 6304-type rolling-element bearings. The waviness profiles were measured at the actual points of contact between the balls and the raceways. The analysis of the literature indicates that there are hardly any works dealing with the determination of the actual points of contact between the bearing surfaces. Therefore, the measurement of the waviness deviation in this location is very important, from the functional point of view.

The waviness deviations were examined in the range of 16-50 UPR. The vibrations generated by deep groove ball bearings were analyzed in three frequency ranges: low LB $(50-300 \mathrm{~Hz})$, medium MB (300-1800 Hz), high HB (1800-10000 Hz), and in addition, in full range RMS. The paper has also presented a procedure for the determination of the points of contact between the ball and the bearing raceways in order to define the location of waviness measurement.

The test results presented in this paper indicate that an increase in the waviness deviations RONt of both the inner and outer raceways of 6304-type single-row deep groove ball bearings causes an increase in the vibration level. This effect is most visible for the medium vibration frequency range $\mathrm{MB}$, and least visible for the low frequency range LB. Furthermore, analyzing the Pearson linear correlation coefficients, it can be concluded that the correlation relationship between the waviness deviation measured on the outer raceways and the medium vibration frequency is greater than for the inner raceways. It should be noted that the values of the Pearson linear correlation coefficients, obtained for the full vibration frequency bandwidth RMS, and the waviness deviation of both the internal and external races are very similar. This indicates a significant correlation between the full vibration frequency range and the waviness deviation of bearing raceways. Therefore, for quantitative impact analysis of waviness deviation and vibration level generated by rolling-element bearing, the full range of vibration RMS should be analyzed.

Considering the vibration measurement, it can also be concluded that the highest values of vibration were obtained in the medium frequency bandwidth MB. Vibration amplitudes noted in the MB range, measured using an Anderometer at a rotation speed equal $1800 \mathrm{~min}^{-1}$, usually result from surface defects of bearing raceways. These defects can be the result of vibration during processing, for example caused by the use of an unbalanced grinding well or wear machining. What is more, the vibration amplitudes measured in the frequency range from 300 to $1800 \mathrm{~Hz}$ arise due to dirt located inside rolling bearings during their exploitation. Based on the research results presented in this paper, it can be concluded that the manufacturing process of examined bearings is not completely stable.

Analyzing the results of waviness measurement it can be noted that the outer races showed greater values of waviness deviation than the inner races. However, these values are still very similar. It has been confirmed through harmonic analysis of vibration velocity signal.

The study results presented in this paper will be the basis for a larger research project aimed at assessing the effect of the geometric structure parameter of active bearing surfaces on the vibration level. In future studies, the authors will conduct detailed analysis of the harmonic components of the waviness profile. Then, the correlation between the individual waviness harmonic components and the vibration components will be obtained. It will allow to develop a diagnostic tool based on non-invasive vibration measurement to determine the approximate values of waviness of the active rolling-element bearing surface. Furthermore, in future studies, a modal analysis of tested bearing will be carried out in order to in detail analyze bearing condition with different defects. Moreover, wider groups of rolling-element bearings (of other types and manufacturers) will be examined, which should give more detailed information on the analyzed diagnostic method.

\section{REFERENCES}

[1] K. Stępień, "In situ measurement of cylindricity - problems and solution", Precision Engineering 38 (3), 697-701 (2015).

[2] S. Adamczak, P. Zmarzły, and D. Janecki, "Theoretical and practical investigations of V-block waviness measurement of cylindrical parts", Metrology and Measurement Systems 22 (2), 181-192 (2015).

[3] Y. Zhuo, X. Zhou, and C. Yang, "Dynamic analysis of double-row self-aligning ball bearings due to applied loads, internal clearance, surface waviness and number of balls", Journal of Sound and Vibration 333 (23), 6170-6189 (2014).

[4] Z. Ren, J. Wang, F. Guo, and A.A. Lubrecht, "Experimental and numerical study of the effect of raceway waviness on the oil film in thrust ball bearings", Tribology International 73, 1-9 (2014). 
[5] I. El-Thalji and J. Erkki, "A summary of fault modelling and predictive health monitoring of rolling element bearings", $\mathrm{Me}$ chanical Systems and Signal Processing 60-61, 252-272 (2015).

[6] J. Suna, R.J.K. Wood, L. Wanga, I. Care, and H.E.G. Powrie, "Wear monitoring of bearing steel using electrostatic and acoustic emission techniques", Wear 259, 1482-1489 (2005).

[7] N. Tandon and A. Choudhury, "A review of vibration and acoustic measurement methods for the detection of defects in rolling element bearings", Tribology International 32, 469-480 (1999).

[8] A. Wang and J. Wang, "Temperature distribution and scuffing of tapered roller bearing", Chinese Journal of Mechanical Engineering 27 (6), 1272-1279 (2014).

[9] M. Li , MQ. Jing, ZF. Chen, and H. Liu, "An improved ultrasonic method for lubricant-film thickness measurement in cylindrical roller bearings under light radial load", Tribology International 78, 35-40 (2014).

[10] L. Zhen, H. Zhengjia, Z. Yanyang, and C. Xuefeng, "Bearing condition monitoring based on shock pulse method and improved redundant lifting scheme", Mathematics and Computers in Simulation 79, 318-338 (2008).

[11] S. Raadnui and S. Kleesuwan, "Electrical pitting wear debris analysis of grease-lubricated rolling element bearings", Wear 271, 1707-1718 (2011).

[12] B. Blachowski, A. Swiercz, P. Gutkiewicz, J. Szelążek, and W. Gutkowski, "Structural damage detectability using modal and ultrasonic approaches", Measurement 85, 210-221 (2016).

[13] Z. Stanik, "Vibro-acoustic diagnostics of rolling bearings in vessels", Trans. Marit. Sci. 3 (2), 111-118 (2014).

[14] E. Miko and Ł. Nowakowski, "Vibrations in the machining system of the vertical machining center", Procedia Engineering 39, 405-413 (2012).

[15] C. Kundera, "Determination and analysis of cross-couplings of axial and angular vibrations of a flexibly mounted ring in a non-contacting face seal", Journal of Tribology - Transactions of the ASME 125 (4), 797-803 (2003).

[16] R. Akhand and S.H. Upadhyay, "A review on signal processing techniques utilized in the fault diagnosis of rolling element bearings", Tribology International 96, 289-306 (2016).

[17] T. Karacay and N. Akturk, "Experimental diagnostics of ball bearings using statistical and spectral methods", Tribology International 42, 836-843 (2009).

[18] J.P. Dron, F. Bolaers, and L. Rasolofondraibe, "Improvement of the sensitivity of the scalar indicators (crest factor, kurtosis) using a de-noising method by spectral subtraction: application to the detection of defects in ball bearings", Journal of Sound and Vibration 270, 61-73(2004)
[19] T.J. Harvey, R.J.K. Wood, and H.E.G. Powrie, "Electrostatic wear monitoring of rolling element bearings", Wear 263, 1492-1501 (2007).

[20] K. Stępień and W. Makieła, "An analysis of deviations of cylindrical surfaces with the use of wavelet transform", Metrology and Measurement Systems 20 (1), 139-150 (2013).

[21] S. Prabhakar, A.R. Mohanty, and A.S. Sekha, "Application of discrete wavelet transform for detection of ball bearing race faults", Tribology International 35, 793-800 (2002).

[22] R.B. Randall, J. Antoni, and S. Chobsaard, "The relationship between spectral correlation and envelope analysis in the diagnostics of bearing faults and other cyclostationary machine signals", Mechanical Systems and Signal Processing 15 (5), 945-962 (2001).

[23] M. Tiwari, K. Gupta, and O. Prakash, "Effect of radial internal clearance of ball bearing on the dynamics of a balanced horizontal rotor", Journal of Sound and Vibration 238, 723-756 (2000).

[24] R.K. Purohit and K. Purohit, "Dynamic analysis of ball bearings with effect of preload and number of balls", Int. J. of Applied Mechanics and Engineering 11 (1), 77-91 (2006).

[25] W. Liu, Y. Zhang, Z.-J. Feng, J.-S. Zhao, and D. Wang, "A study on waviness induced vibration of ball bearings based on signal coherence theory", Journal of Soundand Vibration 333, 6107-6120 (2014).

[26] C.K. Babu, N. Tandon, and R.K. Pandey, "Vibration modeling of a rigid rotor supported on the lubricated angular contact ball bearings considering six degrees of freedom and waviness on balls and races", Journal of Vibration and Acoustics 134 (1), 011006 (2012).

[27] S. Adamczak, P. Zmarzły, and K. Stępień, "Identification and analysis of optimal method parameters of the V-block waviness measurements", Bull. Pol. Ac.: Tech. 64 (2), 45-52 (2016).

[28] R. Kostek, "Simulation and analysis of vibration of rolling bearing”, Key Engineering Materials 588, 257-265 (2013).

[29] S. Adamczak, R. Domagalski, E. Sender, P. Zmarzły, and Ł. Gorycki, "Research methods and testing stand developed to examine vibrations generated by rolling bearing", Diagnostyka 17 (1), 41-49 (2016).

[30] NSK Motion \& Control, Technical Report CAT. No. E728g, 2013, (prospect).

[31] S. Adamczak, A. Janusiewicz, W. Makieła, and K. Stępień, "Statistical validation of the method for measuring radius variations of components on the machine tool”, Metrol. Meas. Syst. 18 (1), 35-46 (2011).

[32] B.P. Graney and K. Starry, "Rolling element bearing analysis", Materials Evaluation 70 (1), 78-85 (2011). 